\title{
Atomic-scale structure and band-gap bowing in $\mathrm{Cu}(\mathrm{In}, \mathrm{Ga}) \mathrm{Se}_{2}$
}

\author{
C. S. Schnohr, ${ }^{1}$ H. Kämmer, ${ }^{1}$ C. Stephan, ${ }^{2}$ S. Schorr, ${ }^{2}$ T. Steinbach, ${ }^{1}$ and J. Rensberg ${ }^{1}$ \\ ${ }^{1}$ Institut für Festkörperphysik, Friedrich-Schiller-Universität Jena, Max-Wien-Platz 1, 07743 Jena, Germany \\ ${ }^{2}$ Helmholtz-Zentrum Berlin für Materialien und Energie, Hahn-Meitner-Platz 1, 14109 Berlin, Germany
}

(Received 16 January 2012; revised manuscript received 10 May 2012; published 6 June 2012)

\begin{abstract}
Mixed systems such as the $\mathrm{Cu}(\mathrm{In}, \mathrm{Ga}) \mathrm{Se}_{2}$ chalcopyrite semiconductor consist of different local atomic arrangements, that is, of different combinations of first-nearest-neighbor cations surrounding the Se anions. The anion position of $\mathrm{Cu}-\mathrm{III}-\mathrm{VI}_{2}$ compounds is predicted to strongly influence the material band gap. We therefore used extended x-ray absorption fine structure spectroscopy to study the atomic-scale structure of $\mathrm{Cu}(\mathrm{In}, \mathrm{Ga}) \mathrm{Se}_{2}$ as a function of composition. Based on these results, the anion position was modeled for all first-nearest-neighbor configurations using a valence force-field approach. We show that the atomic-scale structure strongly depends on the kind of first-nearest-neighbor atoms. Structural relaxation of the anion occurs with respect to both (i) $\mathrm{Cu}$ and group III atoms and (ii) In and $\mathrm{Ga}$ atoms. In both cases, the average anion displacement exhibits a nonlinear behavior with changing composition and thus results in two separate but significant contributions to the band gap bowing observed in $\mathrm{Cu}(\mathrm{In}, \mathrm{Ga}) \mathrm{Se}_{2}$.
\end{abstract}

DOI: 10.1103/PhysRevB.85.245204

PACS number(s): 71.20.Nr, 61.66.Dk, 61.05.cj, 68.35.bd

\section{INTRODUCTION}

Similar to cubic $(A, B) C$ zincblende alloys, mixed $(A, B)(C, D) E_{2}$ chalcopyrite systems allow the tuning of their properties by adjusting the $A$-to- $B$ or $C$-to- $D$ ratio. That way they provide a large and versatile semiconductor material basis for advanced technological applications. $\mathrm{Cu}(\mathrm{In}, \mathrm{Ga}) \mathrm{Se}_{2}$ and $\mathrm{Cu}(\mathrm{In}, \mathrm{Ga}) \mathrm{S}_{2}$, in particular, have received much attention over the last years due to their high potential as absorber materials in photovoltaic applications. Indeed, among the materials used for thin film solar cells, $\mathrm{Cu}(\mathrm{In}, \mathrm{Ga}) \mathrm{Se}_{2}$ currently yields the highest efficiencies with a record value above $20 \%$ on laboratory scale. ${ }^{1}$ However, the chalcopyrite crystal structure (space group $I \overline{4} 2 d$ ) is inherently more complex than the zincblende crystal structure (space group $F \overline{4} 3 \mathrm{~m}$ ) due to the existence of two distinct cation sites. For $\mathrm{CuInSe}_{2}$ and $\mathrm{CuGaSe}_{2}$, the $\mathrm{Cu}$ atoms are located on the Wyckoff position $4 a$ while the group III atoms occupy the Wyckoff position $4 b$ as shown schematically in Fig. $1 .^{2}$ As a consequence, the anion position (Wyckoff position $8 d$ ) may deviate from the ideal lattice site of $(0.25,0.25,0.125)$ due to the different properties of the neighboring cations. Theoretical studies predict a strong influence of this anion position on the semiconductor band gap, which is a fundamental material property for any technological

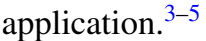

The mixed $\mathrm{Cu}(\mathrm{In}, \mathrm{Ga}) \mathrm{Se}_{2}$ crystallizes in the chalcopyrite structure over the whole compositional range. Yet the random occupation of the $4 b$ position by In and Ga atoms leads to the coexistence of different first-nearest-neighbor (first-NN) configurations surrounding the Se anions as shown in Fig. 1. In contrast, each of the cations is surrounded by four Se anions. It is well known for the simpler (In, Ga)As and (In, Ga)P zincblende alloys that the anion position depends sensitively on the first-NN In and Ga arrangement. ${ }^{6-9}$ The anion position in the mixed $\mathrm{Cu}(\mathrm{In}, \mathrm{Ga}) \mathrm{Se}_{2}$ system will thus be influenced not only by the competition between $\mathrm{Cu}$ and group III atoms but also by the competition between In and Ga atoms. Given the remarkable influence of the anion position on the band gap, we have therefore studied the atomic-scale structure of $\mathrm{Cu}(\mathrm{In}, \mathrm{Ga}) \mathrm{Se}_{2}$ as a function of composition using extended x-ray absorption fine structure spectroscopy (EXAFS). Based on these experiments, the different first-NN configurations have been modeled with a valence force-field approach. The resulting anion positions are analyzed in terms of structural relaxation with respect to (i) $\mathrm{Cu}$ and group III atoms and (ii) In and $\mathrm{Ga}$ atoms. The associated changes of the band gap are estimated and clearly demonstrate that both relaxation effects contribute significantly to the nonlinear change of the band gap with composition known as band gap bowing.

\section{X-RAY ABSORPTION SPECTROSCOPY STUDY}

\section{A. Experimental details}

$\mathrm{Cu}(\mathrm{In}, \mathrm{Ga}) \mathrm{Se}_{2}$ powder samples with different $\mathrm{In} /(\mathrm{In}+\mathrm{Ga})=\mathrm{In} / \mathrm{III}$ and $\mathrm{Cu} /(\mathrm{In}+\mathrm{Ga})=\mathrm{Cu} / \mathrm{III}$ ratios were synthesized by solid state reaction from the pure elements. ${ }^{10-12}$ All samples were prepared in sealed, evacuated silica tubes at a maximum temperature of $850^{\circ} \mathrm{C}$. Several annealing steps with subsequent mechanical grinding were performed to ensure chemical and structural homogeneity. The elemental composition was determined using wavelength dispersive $\mathrm{x}$-ray analysis. ${ }^{11,12}$ To that end, part of the powder was cast in epoxy and mechanically polished to yield a clean and very flat surface. The In/III and $\mathrm{Cu} / \mathrm{III}$ ratios are summarized in Table I. ${ }^{13}$

EXAFS measurements of the $\mathrm{Cu}, \mathrm{Ga}$, and In $K$ edges were performed in transmission mode on $\mathrm{BN}$-diluted powder samples at Beamline C of HASYLAB, Hamburg, Germany. Spectra were recorded at a temperature of $17 \pm 1 \mathrm{~K}$ to minimize thermal vibrations. Aligning the scans with respect to a reference foil or compound measured simultaneously, no significant shift of the edge position with sample composition was observed. The data were processed and analyzed using the IFEFFIT code $^{14}$ and the corresponding user interfaces ATHENA and ARTEMIS. ${ }^{15}$ After background subtraction, the spectra were Fourier transformed over a photoelectron wave number range of $k=3-15 \AA^{-1}$. Figure 2(a) plots the $k^{2}$-weighted EXAFS spectra measured at the In $K$ edge for sample A (In/III = 1.00) and sample $\mathrm{F}(\mathrm{In} / \mathrm{III}=0.50)$ as a function of $k$. The high quality of the data is readily apparent. The corresponding 


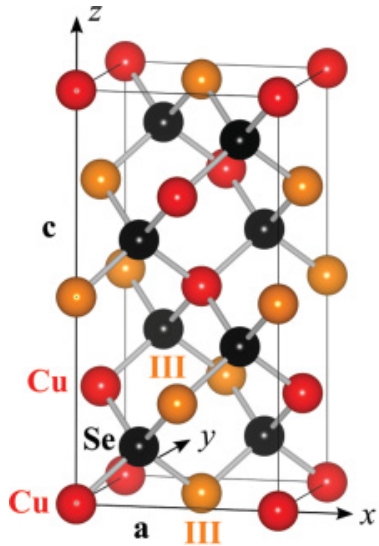

(C1)

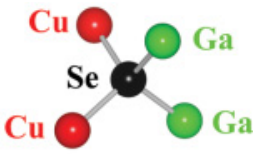

(C2)

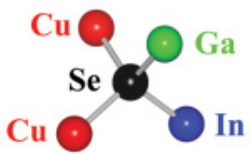

(C3)

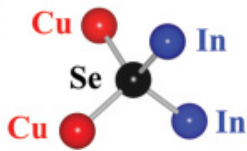

FIG. 1. (Color online) Schematic of the chalcopyrite crystal structure of $\mathrm{Cu}(\mathrm{In}, \mathrm{Ga}) \mathrm{Se}_{2}$ (Ref. 2). The small images on the right illustrate the three different first-NN configurations surrounding the Se anions, namely (C1) two $\mathrm{Cu}$ and two $\mathrm{Ga}$; (C2) two $\mathrm{Cu}$, one $\mathrm{Ga}$, and one In; and (C3) two $\mathrm{Cu}$ and two In. Note that for configuration (C2) the $\mathrm{Ga}$ atom can be either the top or the bottom group III atom.

Fourier transforms are shown in Fig. 2(b). The strong peak at $R \sim 2.0-2.5 \AA$ stems from scattering of the photoelectron wave at first-NN Se atoms while the features at $R \sim 3-5 \AA$ originate from scattering at second- and third-NN atoms and from multiple scattering events.

The first-NN scattering contribution was fitted over a radial range of $R=1.4-2.9 \AA$ for the $\mathrm{Cu}$ and $\mathrm{Ga} K$ edge and $R=$ 1.5-3.0 $\AA$ for the In $K$ edge using multiple $k$ weights of 2, 3, and 4 . Phase shifts and scattering amplitudes were calculated $a b$ initio using FEFF9 (Ref. 16). The amplitude reduction factor $S_{0}{ }^{2}$ and the threshold energy $E_{0}$ were fixed to average values determined from all samples. The mean value $d$ (elementspecific bond length) and the standard deviation $\sigma^{2}$ of the first-NN distance distribution were varied in the fit. In contrast, the coordination number was fixed to four and the asymmetry parameter was kept zero given the very low temperature of $17 \mathrm{~K}^{17}$ The Fourier transformation of the experimental data together with the best fit are shown exemplarily for sample $\mathrm{F}$ $(\mathrm{In} / \mathrm{III}=0.50)$ in Fig. 2(c).

TABLE I. Sample composition given by the $\mathrm{In} / \mathrm{III}=\mathrm{In} /(\mathrm{In}+\mathrm{Ga})$ and $\mathrm{Cu} / \mathrm{III}=\mathrm{Cu} /(\mathrm{In}+\mathrm{Ga})$ ratios determined from wavelength dispersive $\mathrm{x}$-ray analysis (uncertainty \pm 0.01 ). The element-specific bond lengths measured at $17 \mathrm{~K}$ by EXAFS at the $\mathrm{Cu}, \mathrm{Ga}$, and $\mathrm{In} K$ edges for $\mathrm{Cu}(\mathrm{In}, \mathrm{Ga}) \mathrm{Se}_{2}$ are also listed (uncertainty $\pm 0.002 \AA$ ).

\begin{tabular}{lccccc}
\hline \hline Sample & $\mathrm{In} / \mathrm{III}$ & $\mathrm{Cu} / \mathrm{III}$ & $d_{\mathrm{Cu}-\mathrm{Se}}(\AA)$ & $d_{\mathrm{Ga}-\mathrm{Se}}(\AA)$ & $d_{\text {In-Se }}(\AA)$ \\
\hline $\mathrm{A}$ & 1.00 & 0.95 & 2.426 & & 2.584 \\
$\mathrm{~B}$ & 1.00 & 0.84 & 2.425 & & 2.584 \\
$\mathrm{C}$ & 0.91 & 1.08 & 2.425 & 2.433 & 2.583 \\
$\mathrm{D}$ & 0.75 & 0.92 & 2.423 & 2.421 & 2.580 \\
$\mathrm{E}$ & 0.73 & 0.79 & 2.423 & 2.419 & 2.579 \\
$\mathrm{~F}$ & 0.50 & 0.87 & 2.421 & 2.416 & 2.576 \\
$\mathrm{G}$ & 0.25 & 0.88 & 2.417 & 2.413 & 2.573 \\
$\mathrm{H}$ & 0.18 & 1.11 & 2.417 & 2.412 & 2.568 \\
$\mathrm{I}$ & 0.10 & 1.10 & 2.415 & 2.410 & 2.561 \\
$\mathrm{~K}$ & 0 & 0.99 & 2.413 & 2.408 & \\
$\mathrm{~L}$ & 0 & 0.81 & 2.412 & 2.408 & \\
\hline \hline
\end{tabular}
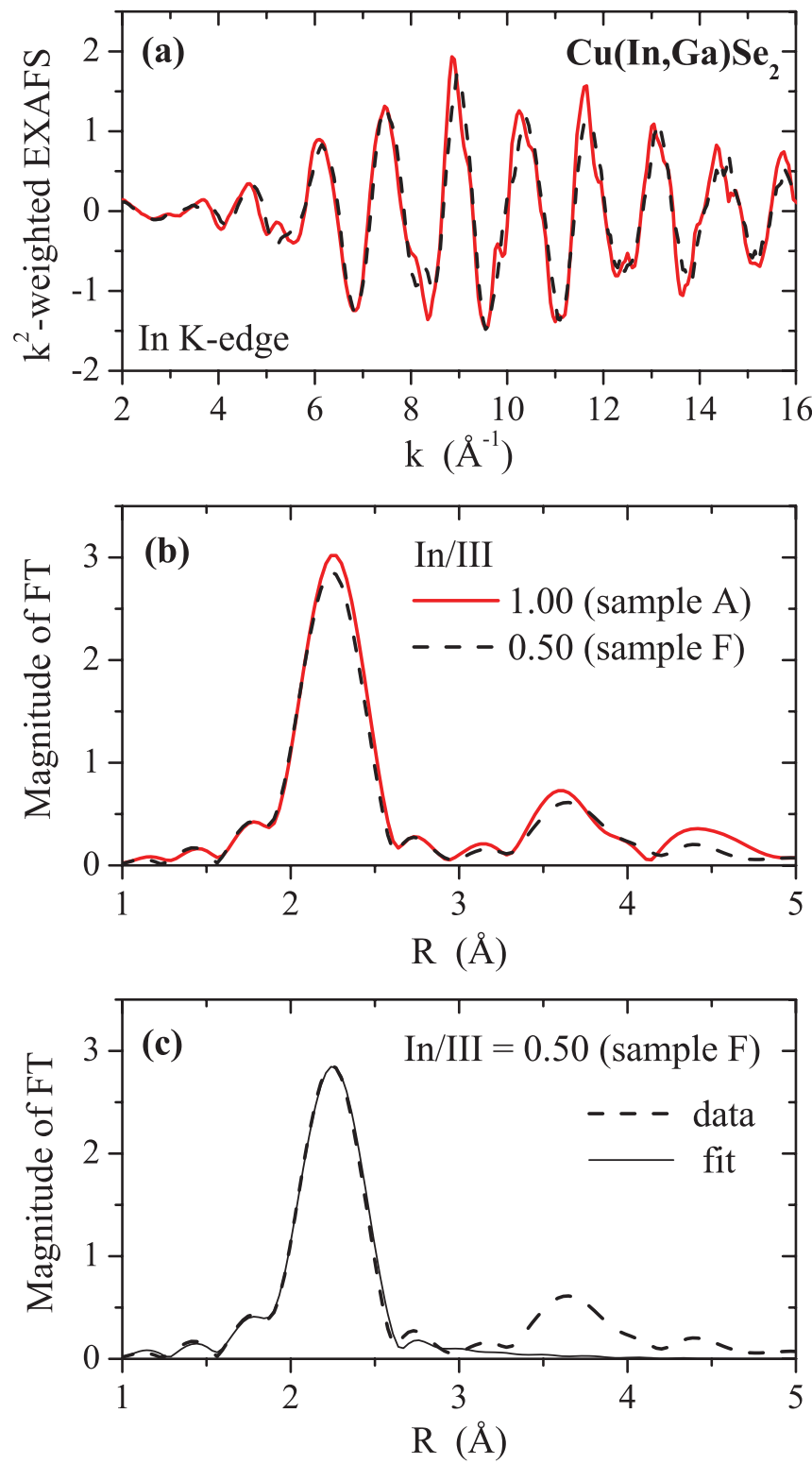

FIG. 2. (Color online) (a) $k^{2}$-weighted EXAFS spectra of $\mathrm{Cu}(\mathrm{In}, \mathrm{Ga}) \mathrm{Se}_{2}$ with $\mathrm{In} / \mathrm{III}=1.00$ (sample A, thick solid line) and 0.50 (sample F, thick dashed line) measured at the In $K$ edge versus photoelectron wave number $k$. (b) Corresponding Fourier transforms (FT) as a function of the radial distance $R$ from the absorber. (c) FT data (thick dashed line) and fit to the first-NN scattering contribution (thin solid line) versus radial distance $R$ for $\mathrm{In} / \mathrm{III}=0.50$ (sample F).

\section{B. First-nearest-neighbor distances}

The element-specific bond lengths $d_{\mathrm{Cu}-\mathrm{Se}}, d_{\mathrm{Ga}-\mathrm{Se}}$, and $d_{\mathrm{In}-\mathrm{Se}}$ are listed in Table I and plotted in Fig. 3 as a function of In/III ratio. The corresponding $\sigma^{2}$ values (not shown) range from 2.5 to $3.9 \times 10^{-3} \AA^{2}$, typical for low temperature measurements. For $\mathrm{CuGaSe}_{2}$, we determined $d_{\mathrm{Cu}-\mathrm{Se}}$ and $d_{\mathrm{Ga}-\mathrm{Se}}$ to be $2.413 \pm 0.002 \AA$ and $2.408 \pm 0.002 \AA$, respectively, corresponding to an anion position of $x=0.2508 \pm 0.0006$ and a respective anion displacement $u=0.25-x=-0.0008 \pm$ 0.0006 , in good agreement with previous reports. ${ }^{3,12}$ For $\mathrm{CuInSe}{ }_{2}$, we determined $d_{\mathrm{Cu}-\mathrm{Se}}$ and $d_{\mathrm{In}-\mathrm{Se}}$ to be $2.426 \pm$ $0.002 \AA$ and $2.584 \pm 0.002 \AA$, respectively, corresponding 


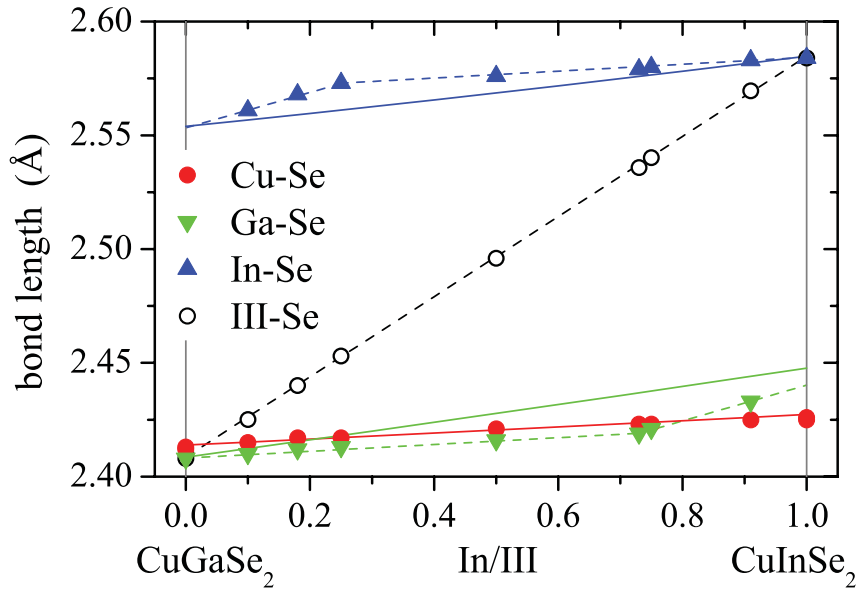

FIG. 3. (Color online) Element-specific bond lengths measured at the $\mathrm{Cu}, \mathrm{Ga}$, and In $K$ edges (solid symbols) as a function of the $\mathrm{In} / \mathrm{III}$ ratio for $\mathrm{Cu}(\mathrm{In}, \mathrm{Ga}) \mathrm{Se}_{2}$. The III-Se bond length (open symbols) is calculated as the weighted average of the Ga-Se and In-Se bond lengths. The dashed lines are guides for the eye, while the solid lines represent the element-specific average first-NN distances obtained from the valence force-field model.

to $x=0.2262 \pm 0.0007$ and $u=0.0238 \pm 0.0007$, again in good agreement with previous studies..$^{3,12,18,19}$ Interestingly, no effect of the $\mathrm{Cu}$ content on the mean first-NN distances is observed for single-phase chalcopyrite samples with $0.80<$ $\mathrm{Cu} / \mathrm{III}<1.00$ (compare samples $\mathrm{A}$ and $\mathrm{B}$ for $\mathrm{CuInSe}_{2}$ and $\mathrm{K}$ and $\mathrm{L}$ for $\mathrm{CuGaSe}_{2}$ in Table I). This is in contrast to the findings of Merino et al. ${ }^{20,21}$ for $\mathrm{CuInSe}_{2}$, but agrees very well with the reports by Kuwahara et al. ${ }^{18}$ and Yamazoe et al. ${ }^{19}$ also on $\mathrm{CuInSe}$.

Regarding $\mathrm{Cu}(\mathrm{In}, \mathrm{Ga}) \mathrm{Se}_{2}$, the lattice constants $a$ and $c$ of the chalcopyrite phase are known to change linearly with In/III ratio between the values of the ternary compounds (Vegard's law). ${ }^{22}$ However, we measured with EXAFS that the element-specific bond lengths remain close to their ternary values over the whole compositional range where $d_{\mathrm{Cu}-\mathrm{Se}} \sim$ $d_{\text {Ga-Se }}$ while $d_{\text {In-Se }}$ is much larger (see Table I and Fig. 3). A similar behavior has already been observed for the simpler group III-V and II-VI $(A, B) C$ zincblende alloys ${ }^{6-8}$ and can be explained by the fact that bond bending is energetically favored over bond stretching. ${ }^{8,23}$ Apparently, this is also true for the more complex $\mathrm{Cu}(\mathrm{In}, \mathrm{Ga}) \mathrm{Se}_{2}$ chalcopyrite system confirming this behavior to be a characteristic feature of tetrahedrally coordinated systems. As a striking consequence, the shortrange atomic arrangement of $\mathrm{Cu}(\mathrm{In}, \mathrm{Ga}) \mathrm{Se}_{2}$ strongly varies from the average, long-range chalcopyrite structure depending on the nature of the cations involved.

Closer examination of the $\mathrm{Cu}(\mathrm{In}, \mathrm{Ga}) \mathrm{Se}_{2}$ bond lengths reveals a slight increase with increasing In/III ratio (see Fig. 3) while no effect is again observed for the $\mathrm{Cu}$ content (compare samples D and E in Table I). $d_{\mathrm{Ga}-\mathrm{Se}}$ and $d_{\mathrm{In}-\mathrm{Se}}$ further show a "knee" at $\mathrm{Ga} / \mathrm{III}=0.25$ and $\mathrm{In} / \mathrm{III}=0.25$, respectively, which is not observed in case of the simpler group III-V or II-VI $(A, B) C$ zincblende alloys. ${ }^{6-8}$ However, in the dilute limit (i.e., for $\mathrm{In} / \mathrm{III} \rightarrow 1$ in the case of $d_{\mathrm{Ga}-\mathrm{Se}}$ and for In/III $\rightarrow 0$ in the case of $d_{\text {In-Se }}$ ) the behavior is again strikingly similar. For both $d_{\mathrm{Ga}-\mathrm{Se}}$ and $d_{\mathrm{In}-\mathrm{Se}}$ the difference between the value in the ternary compound and the dilute limit amounts to approximately $20 \%$ of the difference between the two ternary values. This is in excellent agreement with the findings for (In,Ga)As (Ref. 6) and (In,Ga)P (Ref. 8), suggesting a fundamental similarity between these mixed cation systems independent of crystal structure and anion species.

The weighted average of $d_{\mathrm{Ga}-\mathrm{Se}}$ and $d_{\mathrm{In}-\mathrm{Se}}$ shows a linear increase with increasing In/III ratio (see $d_{\mathrm{III}-\mathrm{Se}}$ in Fig. 3 ) in perfect accordance with neutron diffraction studies, ${ }^{12}$ which measure the average III-Se bond length. An earlier EXAFS study on $\mathrm{Cu}(\mathrm{In}, \mathrm{Ga}) \mathrm{Se}_{2}$ by Antonioli et al. ${ }^{24}$ found a somewhat different behavior than discussed here. However, the authors obtained the In-Se bond length from a measurement of the Se $K$ edge, where the first-NN signal is a superposition of scattering from $\mathrm{Cu}, \mathrm{Ga}$, and In atoms. The present study is therefore deemed more precise as $d_{\text {In-Se }}$ was determined directly from a measurement of the In $K$ edge where the first-NN signal stems solely from scattering at the surrounding Se atoms.

\section{MODELING OF THE ATOMIC-SCALE STRUCTURE}

The coexistence of different local atomic arrangements presents a fundamental feature of randomly mixed systems such as $\mathrm{Cu}(\mathrm{In}, \mathrm{Ga}) \mathrm{Se}_{2}$. In defect-free chalcopyrite material, ${ }^{25}$ the Se anion is surrounded by two $\mathrm{Cu}$ atoms and two group III atoms. This results in three possible first-NN configurations: (C1) two $\mathrm{Cu}$ and two $\mathrm{Ga}$; $(\mathrm{C} 2)$ two $\mathrm{Cu}$, one $\mathrm{Ga}$, and one In; and (C3) two $\mathrm{Cu}$ and two In (see Fig. 1). As we will show, the atomic-scale structure varies significantly for these configurations. As a consequence and in contrast to the ternary compounds, there is no single $\mathrm{Cu}-\mathrm{Se}, \mathrm{Ga}-\mathrm{Se}$, or In-Se distance in the mixed system and the element-specific bond lengths determined by EXAFS still represent average values over different atomic arrangements. To model the structural parameters of the various configurations we have adopted a valence force-field approach that has already been used to successfully describe randomly mixed $(A, B) C$ zincblende alloys. $^{7-9}$

\section{A. Valence force-field model}

Based on the model proposed by Balzarotti et al., ${ }^{7}$ the individual first-NN distances of each configuration can be calculated in the following way. (i) For each In/III ratio $f$, the lattice constants $a(f)$ and $c(f)$ are given by Vegard's law, that is, as the weighted average of the lattice constants of the ternary compounds. (ii) The cations are located on their ideal lattice sites, that is, on the Wyckoff positions $4 a$ and $4 b$ for $\mathrm{Cu}$ and group III atoms, respectively. (iii) The relative anion position $(x, y, z)$ is obtained for each first-NN configuration by minimizing the sum of the bond-stretching energies,

$$
E_{i}=k_{i}\left[\left(d_{i}\right)^{2}-\left(d_{i}^{\text {nat }}\right)^{2}\right]^{2} / 8\left(d_{i}^{\text {nat }}\right)^{2},
$$

where $k_{i}, d_{i}$, and $d_{i}^{\text {nat }}$ denote the bond stretching force constant, the actual first-NN distance, and the natural bond length, respectively, for each of the four cation-anion pairs $i$. Based on diffraction studies, ${ }^{12,26}$ the lattice constants at $17 \mathrm{~K}$ were estimated as $a=5.60 \AA$ and $c=11.01 \AA$ for $\mathrm{CuGaSe}_{2}$ and as $a=5.77 \AA$ and $c=11.61 \AA$ for $\mathrm{CuInSe}_{2}$. The natural 
bond lengths were taken as the EXAFS values determined for the ternary compounds, that is, $d_{\mathrm{In}-\mathrm{Se}}{ }^{\text {nat }}=2.584 \AA$ and $d_{\mathrm{Ga}-\mathrm{Se}}{ }^{\text {nat }}=2.408 \AA$ (see Table I). Interestingly, the natural bond length for the $\mathrm{Cu}-\mathrm{Se}$ pair, $d_{\mathrm{Cu}-\mathrm{Se}}{ }^{\text {nat }}$, depends on the group III configuration and thus $2.413 \AA, 2.420 \AA$, and $2.426 \AA$ were used for configurations (C1), (C2), and (C3), respectively. The bond-stretching force constants were taken as the average of previously reported values, ${ }^{18,27,28}$ yielding $k_{\mathrm{In}-\mathrm{Se}}=k_{\mathrm{Ga}-\mathrm{Se}}=120 \mathrm{~N} / \mathrm{m}$ and $k_{\mathrm{Cu}-\mathrm{Se}}=80 \mathrm{~N} / \mathrm{m}$.

\section{B. First-nearest-neighbor distances}

The individual first-NN distances were calculated using the approach described above for each of the three configurations (C1), (C2), and (C3) and are shown as a function of In/III ratio in Fig. 4. As expected, the cation-anion first-NN distance varies for the different cation species. It does, however, also depend on the atomic configuration and is distinctly different for (C1), (C2), and (C3). Mixed systems such as $\mathrm{Cu}(\mathrm{In}, \mathrm{Ga}) \mathrm{Se}_{2}$ are thus characterized by severe structural fluctuations on the atomic-scale level. Nevertheless, the increase of the overall lattice constant leads to an increase of the individual firstNN distances with increasing In/III ratio. This increase is more pronounced for $\mathrm{Cu}-\mathrm{Se}$ than for $\mathrm{Ga}-\mathrm{Se}$ or $\mathrm{In}-\mathrm{Se}$ due to the lower bond-stretching force constant of the group I-Se bond compared to the group III-Se bonds. A change of the lattice constant is thus accommodated proportionally more by the softer $\mathrm{Cu}-\mathrm{Se}$ bond than by the stiffer III-Se bonds. The minimum-energy arrangement of a given configuration hence depends on the cation-anion pairs involved, on their natural bond lengths, and on the energy required to shorten or lengthen these bonds.

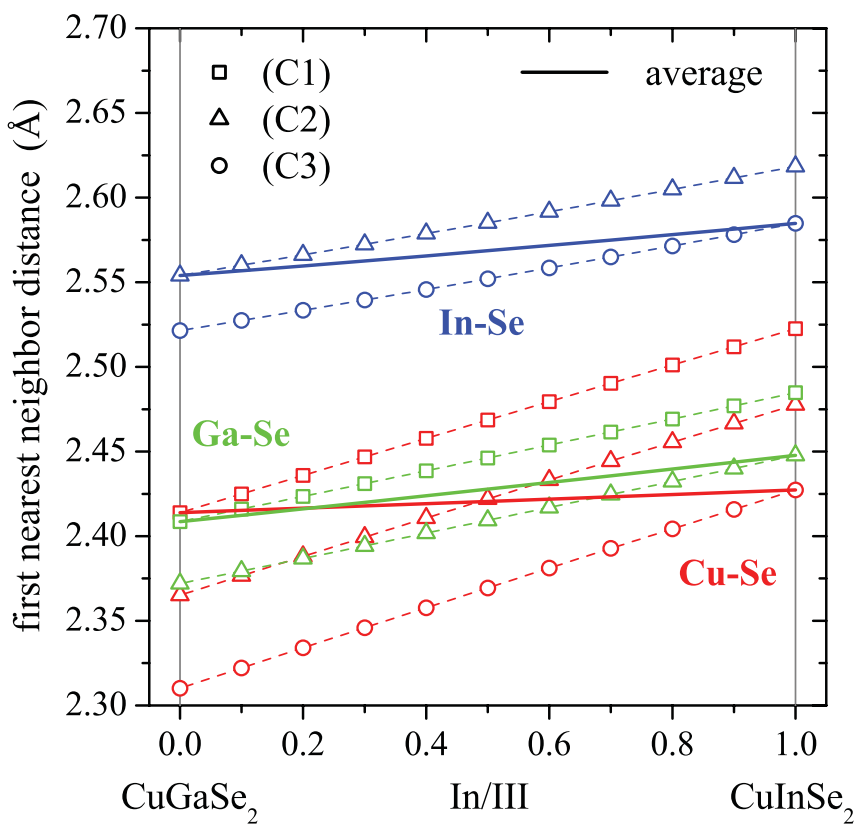

FIG. 4. (Color online) Individual first-NN distances for $\mathrm{Cu}-\mathrm{Se}$ (red), Ga-Se (green), and In-Se (blue) obtained from the valence force-field model for the three different first-NN configurations (C1), (C2), and (C3) versus the $\mathrm{In} / \mathrm{III}$ ratio for $\mathrm{Cu}(\mathrm{In}, \mathrm{Ga}) \mathrm{Se}_{2}$. The resulting element-specific average first-NN distances (element-specific bond lengths) are also given as solid lines.
Assuming a perfectly random distribution of the group III atoms on the $4 b$ Wyckoff position, the probability $P$ for each configuration at a fixed In/III ratio $f$ is given by the binomial distribution: $P^{(C 1)}=(1-f)^{2}, P^{(C 2)}=2 f(1-f)$, and $P^{(C 3)}=f^{2}$. The element-specific mean first-NN distance, corresponding to the EXAFS measurement, can then be obtained as the weighted average over the respective individual first-NN distances of the different configurations. The values are plotted as solid lines in Figs. 3 and 4. As can be seen from Fig. 4, the first-NN distances of the ternary compounds $\mathrm{CuGaSe}_{2}$ and $\mathrm{CuInSe}_{2}$ correspond to those of the configurations (C1) and (C3), respectively, while $d_{\mathrm{Ga}-\mathrm{Se}}$ and $d_{\mathrm{In}-\mathrm{Se}}$ in the dilute limit are given by configuration $(\mathrm{C} 2)$. The increase of the mean first-NN distances with increasing In/III ratio is smaller than that of the individual first-NN distances, demonstrating once more the aspiration of the system to keep the element-specific average cation-anion distances close to their ternary values, that is, close to the natural bond lengths.

Comparing the calculated mean first-NN distances with the experimentally determined element-specific bond lengths (see Fig. 3), excellent agreement is observed for $\mathrm{Cu}-\mathrm{Se}$. Regarding Ga-Se and In-Se, the calculation slightly overand underestimates the experimental values, respectively. In particular, no "knee" is observed in the calculation, which yields a constant linear increase with increasing In/III ratio. Nevertheless, calculated and measured values extrapolated to the dilute limit agree well for In-Se, while for $\mathrm{Ga}-\mathrm{Se}$ the calculation again slightly overestimates the experimental result. A similar effect was previously observed for (In,Ga)P. 8,9 Possible reasons for the differences between calculated and measured values could include (i) deviations from a perfectly random distribution of the group III atoms, ${ }^{29}$ (ii) compositional fluctuations as observed by Yan et al. ${ }^{30}$ together with second-NN interactions (cation relaxation), and (iii) charge redistribution between the Se anion and the three different cation species. Particularly, this latter effect is expected to play an important role as a strong interplay between the first-NN distance and the charge distribution was predicted by Jaffe and Zunger. ${ }^{3}$ The difference between the natural $\mathrm{Cu}-\mathrm{Se}$ bond length in $\mathrm{CuGaSe}_{2}$ and $\mathrm{CuInSe}_{2}$ (see above) also hints at the influence of the charge distribution. Still, given the simplicity of the model, the experimental findings are well described. The model is thus applicable not only to the simpler cubic zincblende systems but also to more complex systems for example with chalcopyrite or kesterite crystal structure. Therefore, it can be used to study the effects of the atomic-scale structure, in particular the anion position, on other material properties such as the band gap.

\section{Anion displacement}

It is well known that in the chalcopyrite crystal structure the anion is typically displaced from its ideal lattice site in the $x$ direction due to the different properties of the neighboring group I and group III cations (see Fig. 1). The natural bond lengths for $\mathrm{Cu}-\mathrm{Se}$ and $\mathrm{Ga}-\mathrm{Se}$ are very similar, leading to an anion position close to the ideal lattice site for $\mathrm{CuGaSe}_{2}$. In contrast, the natural bond length for In-Se is much larger, leading to a significant displacement of the anion toward the $\mathrm{Cu}$ atoms and away from the In atoms in $\mathrm{CuInSe}_{2}$. In the mixed 


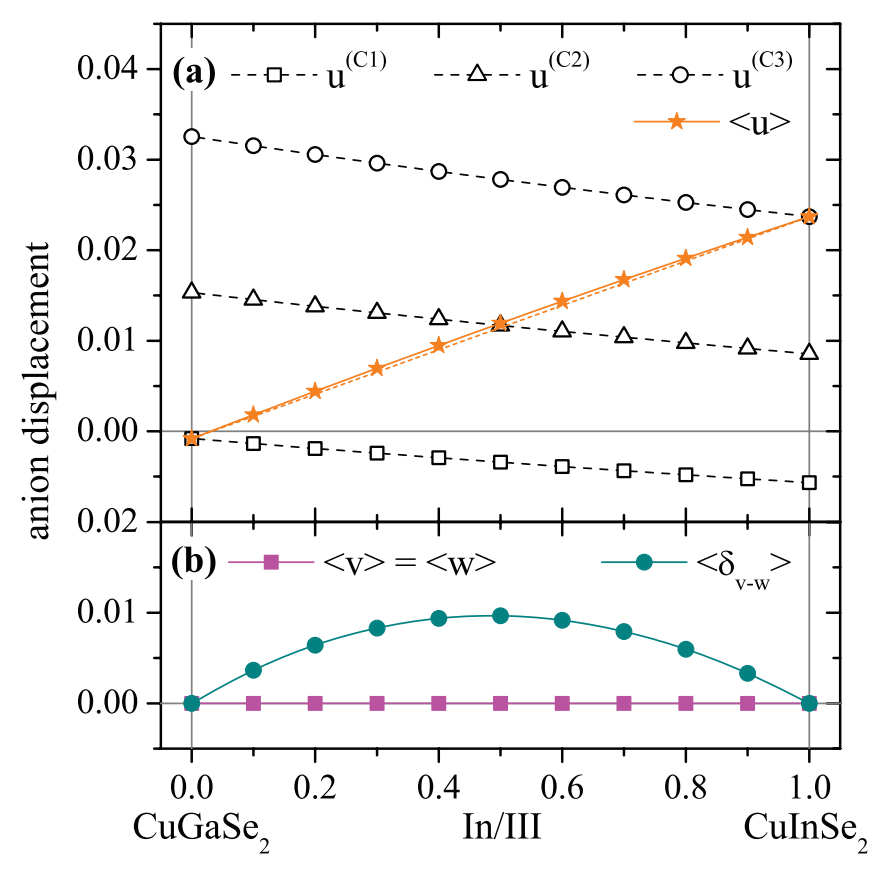

FIG. 5. (Color online) (a) Calculated displacement of the anion in the $x$ direction, $u=0.25-x$, for each of the three different first$\mathrm{NN}$ configurations (C1), (C2), and (C3) versus the In/III ratio for $\mathrm{Cu}(\mathrm{In}, \mathrm{Ga}) \mathrm{Se}_{2}$ (open symbols). The average values $\langle u\rangle$ are also plotted (solid stars). The dotted orange line represents a linear increase for comparison. (b) Calculated average displacement of the anion in the $y$ and $z$ directions, $\langle v\rangle=\langle 0.25-y\rangle$ and $\langle w\rangle=\langle 2(0.125-z)\rangle$, respectively, versus the $\mathrm{In} / \mathrm{III}$ ratio for $\mathrm{Cu}(\mathrm{In}, \mathrm{Ga}) \mathrm{Se}_{2}$. The average displacement from the ideal lattice site within the $y-z$ plane, $\left\langle\delta_{v-w}\right\rangle=$ $\left\langle\sqrt{v^{2}+w^{2}}\right\rangle$, is also given.

$\mathrm{Cu}(\mathrm{In}, \mathrm{Ga}) \mathrm{Se}_{2}$ system, the anion displacement $u=0.25-x$ strongly depends on the local atomic arrangement. Figure 5(a) plots the $u$ values obtained for the configurations (C1), (C2), and (C3) from the model calculation versus the In/III ratio. The values are smallest for $(\mathrm{C} 1)$, intermediate for $(\mathrm{C} 2)$, and largest for (C3). As a consequence, there is no single anion displacement $u$ and the mixed $\mathrm{Cu}(\mathrm{In}, \mathrm{Ga}) \mathrm{Se}_{2}$ is characterized by the coexistence of different anion positions $x$ within the material. The individual values $u$ slightly decrease with increasing In/III ratio because the softer $\mathrm{Cu}-\mathrm{Se}$ bonds expand proportionally more than the stiffer Ga-Se or In-Se bonds, as discussed above. In contrast, the average value $\langle u\rangle$ monotonically increases from approximately zero for $\mathrm{CuGaSe}_{2}$ to almost 0.024 for $\mathrm{CuInSe}_{2}$. Most importantly, however, this increase shows a slight upward bowing, as can be seen by comparison with the dotted line, which represents a linear increase. Albeit very small, this nonlinearity has a significant influence on the material band gap as shown in Sec. IV.

For the configurations (C1) and (C3), and thus for the ternary compounds, the $y$ and $z$ positions of the anion are those of the ideal lattice site, that is, $y=0.25$ and $z=0.125$, due to the symmetry of the first-NN configuration in these directions (see Fig. 1). The corresponding anion displacements $v=0.25-y$ and $w=2 \eta(0.125-z) \sim 2(0.125-z)$ are hence equal to zero. In contrast, for configuration (C2), the anion is also displaced in the $y-z$ plane due to the unequal natural bond lengths of Ga-Se and In-Se. This leads to $v=-w \neq$
0 with $v$ either positive or negative depending on which of the two group III lattice sites is occupied by the Ga atom. The average $v$ and $w$ values are equal to zero, $\langle v\rangle=\langle w\rangle=$ 0 , since the In and $\mathrm{Ga}$ atoms are randomly distributed and both subconfigurations occur with the same probability. The average displacement from the ideal lattice site within the $y-z$ plane, $\left\langle\delta_{v-w}\right\rangle=\left\langle\sqrt{v^{2}+w^{2}}\right\rangle$, however, does not vanish as shown in Fig. 5(b). In contrast, $\left\langle\delta_{v-w}\right\rangle$ shows a strong bowing with In/III ratio being zero for the ternaries and largest for $\mathrm{In} / \mathrm{III}=0.5$. Relaxation of the anion thus occurs both with respect to $\mathrm{Cu}$ and group III atoms in the $x$ direction and with respect to In and $\mathrm{Ga}$ atoms in the $y-z$ plane. In both cases the average displacement shows a nonlinear behavior with changing In/III ratio, which leads to a nonlinear change of the material band gap as is discussed in the following section.

\section{BAND GAP BOWING}

The band gap energy of $\mathrm{Cu}(\mathrm{In}, \mathrm{Ga}) \mathrm{Se}_{2}$ is well known to change nonlinearly between the values of the ternary compounds. The difference $\Delta E(f)$ between the weighted average of the ternary values and the actual $\mathrm{Cu}(\mathrm{In}, \mathrm{Ga}) \mathrm{Se}_{2}$ band gap is typically characterized in terms of the bowing parameter $b$ by $\Delta E(f)=b f(1-f)$, where $f$ denotes again the In/III ratio. Experimentally determined values for $b$ range from 0.15 to $0.24 \mathrm{eV}$, while $b=0.21 \mathrm{eV}$ was obtained from first-principles band-structure calculation. ${ }^{31-35}$ For the simpler $(A, B) C$ zincblende alloys it was shown that three different contributions to this band gap bowing can be distinguished: (i) a band gap change originating from the volume change associated with the varying lattice constants, (ii) a band gap change caused by charge redistribution between the two different cation species, and (iii) a band gap change due to structural relaxation of the anion from its ideal lattice site. ${ }^{9,36,37}$ We will now show that in mixed chalcopyrites, such as $\mathrm{Cu}(\mathrm{In}, \mathrm{Ga}) \mathrm{Se}_{2}$, the situation is more complicated because two different relaxation mechanisms have to be distinguished.

As discussed above, the average displacement $u$ of the Se anion with respect to $\mathrm{Cu}$ and group III atoms in $\mathrm{Cu}(\mathrm{In}, \mathrm{Ga}) \mathrm{Se}_{2}$ changes nonlinearly with In/III ratio and thus contributes to the band gap bowing. To estimate this contribution, the change of $\Delta E$ with changing anion displacement $u$ is needed. For $\mathrm{CuInSe} \mathrm{I}_{2}$, theoretical predictions of this coefficient $\mathrm{d} \Delta E / \mathrm{d} u^{\mathrm{Cu}-\mathrm{III}}$ range from 12 to $32 \mathrm{eV}^{3-5}$ For $\mathrm{CuGaSe}_{2}$, a similar value to that reported for $\mathrm{CuInSe}_{2}$ is predicted. ${ }^{4} \mathrm{An}$ intermediate coefficient of $\mathrm{d} \Delta E / \mathrm{d} u^{\mathrm{Cu}-\mathrm{III}}=20 \mathrm{eV}$ is hence assumed here for all three first-NN configurations of the mixed $\mathrm{Cu}(\mathrm{In}, \mathrm{Ga}) \mathrm{Se}_{2}$ system. The resulting contribution to the band gap bowing is given by $\Delta E^{\mathrm{Cu}-\mathrm{III}}=\langle u\rangle_{\text {nonlin }} \mathrm{d} \Delta E / \mathrm{d} u^{\mathrm{Cu}-\mathrm{III}}$, where $\langle u\rangle_{\text {nonlin }}$ denotes the nonlinearity of $\langle u\rangle . \Delta E^{\mathrm{Cu}-\mathrm{III}}$ is plotted as a function of In/III ratio in Fig. 6 together with the range of experimentally determined $\Delta E$ values. Even though the nonlinearity of $\langle u\rangle$ is very small, the resulting $\Delta E^{\mathrm{Cu}-\mathrm{III}}$ is clearly significant due to the strength of the coefficient $\mathrm{d} \Delta E / \mathrm{d} u^{\mathrm{Cu}-\mathrm{III}}$. As shown recently by Vidal and co-workers, this value of $\mathrm{d} \Delta E / \mathrm{d} u^{\mathrm{Cu}-\mathrm{III}}$ strongly depends on the details of the theoretical study performed. 5 They obtained values of approximately 13, 20, and $32 \mathrm{eV}$ using several different approaches. Since this coefficient is directly multiplied with $\langle u\rangle_{\text {nonlin }}$, the resulting band gap bowing $\Delta E^{\mathrm{Cu}-\mathrm{III}}$ also depends 


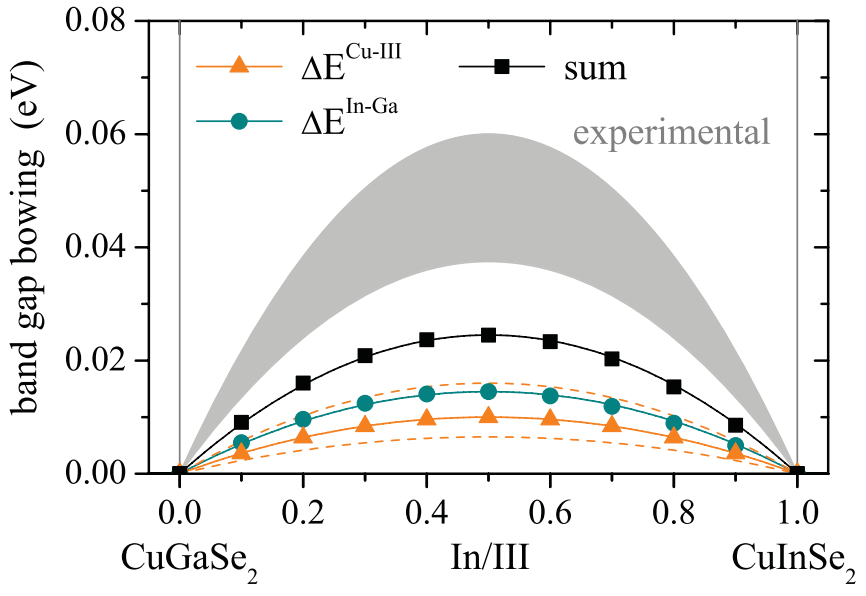

FIG. 6. (Color online) Energy difference $\Delta E$ between the weighted average of the ternary band gaps and the $\mathrm{Cu}(\mathrm{In}, \mathrm{Ga}) \mathrm{Se}_{2}$ band gap versus the In/III ratio. Plotted are the contributions originating from structural relaxation of the anions with respect to $\mathrm{Cu}$ and group III atoms, $\Delta E^{\mathrm{Cu}-\mathrm{III}}$, and with respect to In and $\mathrm{Ga}$ atoms, $\Delta E^{\mathrm{In}-\mathrm{Ga}}$. Their sum and the range of experimentally determined $\Delta E$ values (Refs. 31-35) (shaded area) are also shown. The lower and upper dashed orange lines correspond to $\Delta E^{\mathrm{Cu}-\mathrm{III}}$ calculated with values of 13 and $32 \mathrm{eV}$, respectively, for the coefficient $\mathrm{d} \Delta E / \mathrm{d} u^{\mathrm{Cu}-\mathrm{III}}$, while the solid orange line corresponds to an intermediate value of $20 \mathrm{eV} .^{5}$

on the theoretical approach used. The values obtained with 13 and $32 \mathrm{eV}$ are given by the lower and upper dashed lines in Fig. 6, respectively, and demonstrate the importance of correctly determining the coefficient $\mathrm{d} \Delta E / \mathrm{d} u^{\mathrm{Cu}-\mathrm{III}}$ from theoretical studies. Nevertheless, it is clear that $\Delta E^{\mathrm{Cu}-\mathrm{III}}$ represents a significant contribution to the band gap bowing independent of the exact value of $\mathrm{d} \Delta E / \mathrm{d} u^{\mathrm{Cu}-\mathrm{III}}$.

Band gap bowing is also observed for (In,Ga)P and (In,Ga)As, where the anion relaxation takes place between the $\mathrm{Ga}$ and In first-NN atoms. ${ }^{9,29,36,38}$ The corresponding coefficient $\mathrm{d} \Delta E / \mathrm{d} u^{\mathrm{In}-\mathrm{Ga}}$ was calculated as $1.5 \mathrm{eV}$ and approximately $1.2 \mathrm{eV}$ for $\mathrm{InGaP}_{2}$ (Ref. 36) and (In,Ga)As (Ref. 38), respectively. As discussed above, structural relaxation of the Se anion with respect to $\mathrm{Ga}$ and $\mathrm{In}$ in $\mathrm{Cu}(\mathrm{In}, \mathrm{Ga}) \mathrm{Se}_{2}$ is described by the average displacement $\left\langle\delta_{v-w}\right\rangle$ in the $y-z$ plane. Assuming a similar coefficient $\mathrm{d} \Delta E / \mathrm{d} \delta_{v-w}{ }^{\text {In-Ga }}=1.5 \mathrm{eV}$, this anion displacement between the group III atoms results in a contribution to the band gap bowing given by $\Delta E^{\mathrm{In}-\mathrm{Ga}}=$ $\left\langle\delta_{v-w}\right\rangle \mathrm{d} \Delta E / \mathrm{d} \delta_{v-w}{ }^{\text {In-Ga }}$, which is also plotted in Fig. 6. Here, the relatively small coefficient $\mathrm{d} \Delta E / \mathrm{d} \delta_{v-w}{ }^{\text {In-Ga }}$ is compensated by the strong nonlinear behavior of $\left\langle\delta_{v-w}\right\rangle$, resulting again in a significant contribution to $\Delta E$. Compared to $(\mathrm{In}, \mathrm{Ga}) \mathrm{P}$, however, the effect is approximately a factor of two smaller because the $\mathrm{P}$ anion is surrounded by four group III atoms, leading to larger displacements from the ideal lattice site. ${ }^{9}$ Still, the sum of $\Delta E^{\mathrm{In}-\mathrm{Ga}}$ and $\Delta E^{\mathrm{Cu}-\mathrm{III}}$, and thus the total band gap bowing caused by structural relaxation of the anion in chalcopyrite $\mathrm{Cu}(\mathrm{In}, \mathrm{Ga}) \mathrm{Se}_{2}$, is of similar order as that observed in the zincblende alloy (In, Ga)P.

\section{DISCUSSION}

Similar to mixed $(A, B) C$ zincblende alloys, $\mathrm{Cu}(\mathrm{In}, \mathrm{Ga}) \mathrm{Se}_{2}$ consists of different local atomic arrangements. The situation is, however, more complex, as the chalcopyrite crystal structure differentiates between two distinct cation sites. Structural relaxation of the anion with respect to $\mathrm{Cu}$ and group III atoms is thus a feature inherent to the chalcopyrite structure and occurs in both the ternary compounds and the mixed system. It leads to a displacement in the $x$ direction but not in the $y$ or $z$ direction. In contrast, structural relaxation of the anion with respect to In and $\mathrm{Ga}$ is unique to the mixed system independent of crystal structure. In the chalcopyrites, it leads to a displacement in the $y-z$ plane but not along the $x$ direction. The increased complexity of the chalcopyrite structure compared to the simpler zincblende structure hence results in two distinct structural relaxation effects operative in the mixed system.

The necessity for differentiating the two effects becomes most prominent when studying their influence on the band gap of the material. The average displacement of the anion from its ideal lattice site shows a nonlinear change with the In/III ratio in both cases, which originates from the coexistence of different local atomic arrangements. As we have demonstrated, this nonlinearity is much more pronounced for relaxation between In and $\mathrm{Ga}$ than for relaxation between $\mathrm{Cu}$ and group III atoms. Having said that, the change in band gap associated with a displacement of the anion is much larger for $\mathrm{Cu}$ and group III atoms than for In and $\mathrm{Ga}$ because of the smaller electronegativity difference of the latter. ${ }^{3}$ The resulting band gap bowing, which is a combination of both of these aspects, turns out to be similar in magnitude in both cases and constitutes a significant contribution to the total bowing. Nevertheless, these two structural relaxation effects have to be taken into account separately and independently when studying the interplay between atomic-scale structure, defects caused by off-stoichiometry, and electronic properties for $\mathrm{Cu}(\mathrm{In}, \mathrm{Ga}) \mathrm{Se}_{2}$. While we have demonstrated the occurrence of two distinct relaxation effects for the specific case of $\mathrm{Cu}(\mathrm{In}, \mathrm{Ga}) \mathrm{Se}_{2}$, we believe that it represents a general feature of materials with distinct cation sites. Similar effects are therefore expected to occur for other mixed I-III- $\mathrm{VI}_{2}$ or II-IV-V $V_{2}$ materials, such as $\mathrm{Cu}(\mathrm{Al}, \mathrm{Ga}) \mathrm{S}_{2}$ and $(\mathrm{Cu}, \mathrm{Ag}) \mathrm{InTe}_{2}$ or $\mathrm{Zn}(\mathrm{Si}, \mathrm{Ge}) \mathrm{P}_{2}$ and $(\mathrm{Zn}, \mathrm{Cd}) \mathrm{SnAs}_{2}$, spanning the vast group of chalcopyrite and pnictide materials.

\section{CONCLUSION}

The atomic-scale structure of $\mathrm{Cu}(\mathrm{In}, \mathrm{Ga}) \mathrm{Se}_{2}$ with different $\mathrm{In} / \mathrm{III}$ and $\mathrm{Cu} / \mathrm{III}$ ratios was studied experimentally using EXAFS of the $\mathrm{Cu}, \mathrm{Ga}$, and $\mathrm{In} K$ edges at $17 \mathrm{~K}$. We find that the mean $\mathrm{Cu}-\mathrm{Se}, \mathrm{Ga}-\mathrm{Se}$, and In-Se first-NN distances are very different from each other and remain close to the values of the ternary compounds over the whole compositional range. Based on these results, the different first-NN configurations surrounding the anions in the mixed $\mathrm{Cu}(\mathrm{In}, \mathrm{Ga}) \mathrm{Se}_{2}$ system were modeled using a valence force-field approach. We find that the structural parameters, most prominently the first-NN distances and the anion position, strongly vary depending on the local cation configuration. As a consequence, $\mathrm{Cu}(\mathrm{In}, \mathrm{Ga}) \mathrm{Se}_{2}$ is characterized by severe structural fluctuations on the atomicscale level. Relaxation of the anion from its ideal lattice site occurs with respect to $\mathrm{Cu}$ and group III atoms and with respect to In and $\mathrm{Ga}$ atoms. Both effects are distinctly 
different regarding the direction of relaxation, the magnitude of displacement, and the influence on the band gap and therefore have to be considered separately and independently. The coexistence of different local arrangements in $\mathrm{Cu}(\mathrm{In}, \mathrm{Ga}) \mathrm{Se}_{2}$ leads to a nonlinear change of the average anion displacement with In/III ratio in both cases. For relaxation with respect to $\mathrm{Cu}$ and group III atoms, this nonlinearity is very small but the coefficient describing the change of the band gap with changing anion position is large. In combination, this results in a significant contribution to the experimentally observed band gap bowing. For relaxation with respect to In and $\mathrm{Ga}$ atoms, the average anion displacement exhibits a very strong nonlinear behavior, yet the coefficient describing the effect on the band gap is relatively small. The resulting contribution to the band gap bowing turns out to be of similar order as that originating from relaxation between $\mathrm{Cu}$ and group III atoms. We have clearly demonstrated that the two different relaxation mechanisms present in the mixed $\mathrm{Cu}(\mathrm{In}, \mathrm{Ga}) \mathrm{Se}_{2}$ system result in two individual and significant contributions to the band gap bowing, highlighting the interplay between atomic-scale structural parameters and important material properties. While the study was performed for the specific case of $\mathrm{Cu}(\mathrm{In}, \mathrm{Ga}) \mathrm{Se}_{2}$, we believe that our findings are also applicable to other mixed systems with the chalcopyrite crystal structure.

\section{ACKNOWLEDGMENTS}

The authors thank the HASYLAB staff for their valuable assistance during the measurements. We also thank Carsten Ronning for helpful discussions. This study was financially supported by the Carl-Zeiss-Stiftung, Germany.
${ }^{1}$ P. Jackson, D. Hariskos, E. Lotter, S. Paetel, R. Wuerz, R. Menner, W. Wischmann, and M. Powalla, Prog. Photovolt.: Res. Appl. 19, 894 (2011).

${ }^{2}$ Images created with VESTA; K. Momma, and F. Izumi, J. Appl. Crystallogr. 41, 653 (2008).

${ }^{3}$ J. E. Jaffe and A. Zunger, Phys. Rev. B 29, 1882 (1984).

${ }^{4}$ S.-H. Wei, A. Zunger, I.-H. Choi, and P. Y. Yu, Phys. Rev. B 58, R1710 (1998).

${ }^{5}$ J. Vidal, S. Botti, P. Olsson, J.-F. Guillemoles, and L. Reining, Phys. Rev. Lett. 104, 056401 (2010).

${ }^{6}$ J. C. Mikkelsen Jr., and J. B. Boyce, Phys. Rev. Lett. 49, 1412 (1982).

${ }^{7}$ A. Balzarotti, N. Motta, A. Kisiel, M. Zimnal-Starnawska, M. T. Czyznyk, and M. Podgorny, Phys. Rev. B 31, 7526 (1985).

${ }^{8}$ C. S. Schnohr, L. L. Araujo, P. Kluth, D. J. Sprouster, G. J. Foran, and M. C. Ridgway, Phys. Rev. B 78, 115201 (2008).

${ }^{9}$ C. S. Schnohr, [J. Phys.: Condens. Matter (unpublished)].

${ }^{10}$ C. Stephan, S. Schorr, M. Tovar, and H.-W. Schock, Appl. Phys. Lett. 98, 091906 (2011).

${ }^{11}$ C. Stephan, S. Schorr, T. Scherb, C. A. Kaufmann, and H.-W. Schock, [Appl. Phys. Lett (unpublished)].

${ }^{12}$ C. Stephan, Ph.D. thesis, Freie Universität Berlin/HelmholtzZentrum Berlin, Germany, 2011, http://dx.doi.org/10.5442/d0011.

${ }^{13}$ Note that while electron microprobe analysis did not show any secondary phases for sample $\mathrm{C}$, samples $\mathrm{H}$ and I were found to contain small amounts of $\mathrm{Cu}_{2} \mathrm{Se}$. This additional phase can only influence the measurement at the $\mathrm{Cu} K$ edge but not the spectra recorded at the In or Ga $K$ edge. However, no deviation of samples $\mathrm{H}$ and $\mathrm{I}$ from the overall trend is observed even for $d_{\mathrm{Cu}-\mathrm{Se}}$. This suggests that the amount of $\mathrm{Cu}_{2} \mathrm{Se}$ is small and/or that the average $\mathrm{Cu}$-Se distance in $\mathrm{Cu}_{2} \mathrm{Se}$ is similar to that in the chalcopyrite phase. All samples with $\mathrm{Cu} / \mathrm{III}<1$ were of single chalcopyrite phase.

${ }^{14}$ M. Newville, J. Synchrotron Radiat. 8, 322 (2001).

${ }^{15}$ B. Ravel and M. Newville, J. Synchrotron Radiat. 12, 537 (2005).

${ }^{16}$ J. J. Rehr, J. J. Kas, F. D. Vila, M. P. Prange, and K. Jorissen, Phys. Chem. Chem. Phys. 12, 5503 (2010).

${ }^{17}$ Including a third cumulant in the fit had no significant effect on the $d$ values obtained but strongly increased the parameter uncertainties due to correlation effects.
${ }^{18}$ Y. Kuwahara, H. Oyanagi, H. Yamaguchi, M. Aono, S. Shirakata, and S. Isomura, J. Appl. Phys. 76, 7864 (1994).

${ }^{19}$ S. Yamazoe, H. Kou, and T. Wada, J. Mater. Res. 26, 1504 (2011).

${ }^{20}$ J. M. Merino, J. L. Martín de Vidales, S. Mahanty, R. Díaz, F. Rueda, and M. León, J. Appl. Phys. 80, 5610 (1996).

${ }^{21}$ J. M. Merino, S. Díaz-Moreno, G. Subías, and M. León, Thin Solid Films 480-481, 295 (2005).

${ }^{22}$ M. R. Balboul, H.-W. Schock, S. A. Fayak, A. Abdel El-Aal, J. H. Werner, and A. A. Ramadan, Appl. Phys. A 92, 557 (2008).

${ }^{23}$ C. S. Schnohr, P. Kluth, L. L. Araujo, D. J. Sprouster, A. P. Byrne, G. J. Foran, and M. C. Ridgway, Phys. Rev. B 79, 195203 (2009).

${ }^{24}$ G. Antonioli, S. Bini, P. P. Lottici, and C. Razzetti, J. Phys. 47, 431 (1986).

${ }^{25}$ Defect-free material has been assumed for simplicity as the $\mathrm{Cu} / \mathrm{III}$ ratio does not influence the experimentally determined elementspecific bond lengths (see Sec. II).

${ }^{26}$ W. Paszkowicz, M. Knapp, C. Baehtz, R. Minikayev, P. Piszora, J. Z. Jiang, and R. Bacewicz, J. Alloys Compd. 382, 107 (2004).

${ }^{27}$ P. P. Lottici, G. Antonioli, and C. Razzetti, Phys. Rev. B 37, 9017 (1988).

${ }^{28}$ R. Shioda, Y. Okada, H. Oyanagi, S. Niki, A. Yamada, and Y. Makita, J. Cryst. Growth 150, 1196 (1995).

${ }^{29}$ G. P. Srivastava, J. L. Martins, and A. Zunger, Phys. Rev. B 31, 2561 (1985).

${ }^{30}$ Y. Yan, R. Noufi, K. M. Jones, K. Ramanathan, M. M. Al-Jassim, and B. J. Stanbery, Appl. Phys. Lett. 87, 121904 (2005).

${ }^{31}$ S.-H. Wei and A. Zunger, J. Appl. Phys. 78, 3846 (1995).

${ }^{32}$ K. Yoshino, M. Iwamoto, H. Yokoyama, A. Fikuyama, K. Maeda, S. Niki, and T. Ikari, Jpn. J. Appl. Phys. 38, 3171 (1999).

${ }^{33}$ C. A. Durante Rincón, E. Hernández, M. I. Alonso, M. Garriga, S. M. Wasim, C. Rincón, and M. León, Mater. Chem. Phys. 70, 300 (2001).

${ }^{34}$ P. D. Paulson, R. W. Birkmire, and W. N. Shafarman, J. Appl. Phys. 94, 879 (2003).

${ }^{35}$ S.-H. Han, F. S. Hasoon, J. W. Pankow, A. M. Hermann, and D. H. Levi, Appl. Phys. Lett. 87, 151904 (2005).

${ }^{36}$ A. Zunger and J. E. Jaffe, Phys. Rev. Lett. 51, 662 (1983).

${ }^{37}$ J. E. Bernard and A. Zunger, Phys. Rev. B 36, 3199 (1987).

${ }^{38}$ R. Magri, S. Froyen, and A. Zunger, Phys. Rev. B 44, 7947 (1991). 